\title{
Community-onset bacteremia may be an ominous sign
}

\author{
C. Ruef
}

Published online: 12 October 2011

(C) Springer-Verlag 2011

Several community-acquired infections, such as pneumonia, urinary tract infection, and diarrhea, may be associated with bacteremia in a minority of patients. Bacteremia is considered to be a normal variation of the clinical presentation of such infections and, as such, it generally does not change the physician's approach to therapeutic managment of the infection. However, experienced physicians are clearly aware of the possibility that bacteremia may lead to secondary metastatic complications, such as bacterial arthritis, spondylodiscitis, or endocarditis. Certain pathogens, such as pneumococci, are more frequently associated with such complications than others, such as, for example, Escherichia coli. Good clinical management of these infections requires that the patient be examined at frequent intervals to control the development of such complications and to ensure that the duration of antibiotic therapy is carefully tailored to the clinical course of the specific infection.

Although all experienced infectious disease physicians have been confronted earlier or later in their medical career with infections that have taken a lethal course, a significant risk of a lethal outcome is usually not associated with community-acquired bacteremia within our current conceptual framework of these types of infections. The study by Laupland et al. [1] in this issue of Infection provides the reader with a very interesting insight into short- and longterm mortality associated with community-onset bacteremia. The study is based on data on the Calgary Zone complied in the electronic surveillance system database of

C. Ruef ( $\square)$

Division of Infectious Diseases and Hospital Epidemiology,

HAL 14C, University Hospital of Zurich,

8091 Zurich, Switzerland

e-mail: christian.ruef@usz.ch the Alberta Health Services System. Analysis of this database by the authors revealed that $12 \%$ of 2,448 patients died within 28 days following the diagnosis of communityacquired bacteremia and that one quarter of the patients had died by 1 year post-diagnosis. While these rates reflect all-cause case fatality, they seem to be higher than those most clinicians would expect to experience in their daily practice. Not unexpectedly, older age and significant co-morbidities were independently associated with late mortality. In contrast, bacteremia caused by Streptococcus pneumoniae was not associated with late mortality. While this study does not answer the question of whether early appropriate antibiotic therapy reduces both short- and longterm mortality, the data are nevertheless interesting, showing that patients with bacteremia may have an increased risk of dying within 1 year, especially if underlying diseases, older age, prolonged hospitalization, and polymicrobial infection are present as risk factors.

These data will most likely not change our diagnostic and therapeutic approach to managing patients with suspected bacteremia, but they will help us to have a more sober outlook regarding the prognosis of patients with community-acquired bacteremia, especially when risk factors are present.

C. Ruef, Editor-in-Chief, Infection

\section{Reference}

1. Laupland KB, Svenson LW, Gregson DB, Church DL. Long-term mortality associated with community-onset bloodstream infection. Infection. 2011;39. doi:10.1007/s15010-011-0149-x. 